\title{
ק-Ray Spectroscopy using a Hollow Plastic Scintillator
}

\author{
D. G. GARDNER* and W. W. MEINKE \\ Department of Chemistry, University of Michigan, \\ Ann Arbor, Michigan
}

\begin{abstract}
Specially designed plastic scintillators employing a hollow construction to minimize electron scattering have been used to study accurately $\beta$-spectral distributions down to energies of less than $0 \cdot 1 \mathrm{MeV}$. This inexpensive addition to a scintillation $\gamma$-ray spectrometer makes possible resolution of the $\beta$-spectra of isotopic mixtures. This unit is of particular importance in multiple tracer studies involving pure $\beta$-emitters.
\end{abstract}

\section{LA SPECTROSCOPIE DES RAYONS $\beta$ UTILISANT UN SCINTILLATEUR GREUX EN PLASTIQUE}

Des scintillateurs en plastique particulièrement dessinés ayant une forme de construction creuse, afin de mettre au minimum la dispersion des électrons, a servi à l'étude précise des distributions spectrales de rayons $\beta$ jusqu'à des énergies de moins de $0,1 \mathrm{MeV}$.

Cette addition peu coûteuse à un spectromètre à scintillation pour rayons $\gamma$ rend possible la résolution des spectres $\beta$ des mélanges isotopiques. Cet élément a une importance particulière aux études à indicateurs multiples comportant des émetteurs $\beta$ pures.

\section{ק-СIЕКТРОСКОПИЯ С ПРИМЕНЕНИЕМ ПОЛЫХ СЦИНТИЛЛЯТОРОВ ИЗ ПЛАСТМАССЫ}

Полые пластмассовые сцинтилляторы из особой конструкции, расчитанные на уменышение расселния электронов, были применены для толных измерений $\beta$-спектра при әнергиях ниже 0 , I мәв. Әто недорого стоюцее добавтение к сцинтилляцинному $\gamma$-спектрометру дает возможность разрешать $\beta$-спектры изотопных смесей. Этот прибор особенно пригоден для исстедований, связанных с применением нескольких изотопон, яв.тяющихся чистыми $\beta$-изучателями.

\section{$\beta$-SPECTROSKOPIE MIT HILFE EINES HOHLEN PLASTIKSZINTILLATORS \\ Zur genauen Bestimmung der spektralen Verteilung von $\beta$-Strahlen bis zu Energien niedriger als 0,1 MeV wurde ein besonders konstruierter Szintillator verwendet, dessen innere Höhlung die Streuung der Elektronen auf ein Minimum herabsetzt. Dieser ziemlich billige Ergänzungsteil zu einem Gamma-Spektrometer ermöglicht die Messung von $\beta$-Spektren in Gemischen von verschiedenen Strahlem. Das Gerät ist besonders geeignet für multiple Indikatoruntersuchungen bei welchen reine $\beta$-Strahler verwendet werden.}

In the past few years $\gamma$-ray spectroscopy has become increasingly more common in the industrial and biological research laboratory. $\beta$-ray spectroscopy has not, however, experienced the same growth. This is unfortunate because in many instances $\beta$-spectral information would be preferable to $\gamma$-ray data in the identification and/or assay of radioactive species.

A $\beta$-ray spectrometer would be useful when working with such isotopes as $\mathrm{S}^{\mathbf{3 5}}$ and $\mathrm{P}^{32}$ which do not emit $\gamma$-rays; e.g., the

\footnotetext{
* Present address: Westinghouse Electric Corporation, Pittsburgh, Pa.
} 
amount of $\mathrm{P}^{33}$ in a $\mathrm{P}^{32}$ sample could be determined without the necessity of following a decay curve for months. In experiments with mixed tracers which emit similar $\gamma$-radiations, beta spectral analysis would be an alternate method for obtaining the relative abundance of each tracer. In addition, the $\beta$-spectrum would be another means of characterizing an unknown activity in a radioactive mixture.

The limited availability of simple, low $\operatorname{cost} \beta$-ray spectrometers has restricted their use in the past. This paper describes a type of spectrometer using a hollow scintillation detector which has permitted the study of beta spectral distributions down to energies of less than $0.1 \mathrm{MeV}$. The resolution of this hollow detector is shown to be much better than that of a flat detector of the same scintillation material. Further advantages include simplicity of construction and low cost. The detector operates with a $2 \pi$ geometry in an air atmosphere which facilitates source changing, and requires relatively small amounts of activity.

This spectrometer is sufficiently accurate for most routine needs and represents an inexpensive addition to any scintillation $\gamma$ ray spectrometer.

\section{ß-RAY SPECTROMETERS}

Magnetic spectrometers provide excellent $\beta$-spectral determinations, but they possess certain drawbacks. Their excellent resolution is accompanied by a low transmission which requires high sample activity. Their vacuum system imposes restrictions on sample preparation and ease of sample changing. In addition they have a relatively high cost.

$\beta$-scintillation spectrometers utilize $\beta$-sensitive fluorescent detectors which can be made interchangeable with detectors in a $\gamma$-ray scintillation spectrometer. In the usual arrangement ${ }^{(1,2)}$ the detector is mounted on a photomultiplier tube with the $\beta$-ray source placed at some short distance in front of the detector. This arrangement, however, tends to distort the $\beta$-spectrum since some of the $\beta$-particles will be scattered out of the phosphor, leaving only part of their energy behind. In many cases this leads to significant distortion over the lower two-thirds of the spèctrum.

A method designed to circumvent the scattering effect( ${ }^{(3)}$ requires that the sample be placed within the detector. This method is restricted to pure $\beta$-emitters, since any $\beta$-ray background cannot be subtracted. In addition, energies of radiations emitted in coincidence but at different angles will add, thus producing spurious pulses representing the sum of the energies of these radiations.

In 1950 P. R. BELL suggested an arrangement known as the Hollow Crystal Spectrometer. ${ }^{(3-7)}$ In this instrument the $\beta$-rays are collimated into a conical hole in the phosphor, thus reducing the probability of escape of scattered $\beta$-rays. A modified version of this type of spectrometer has been developed and is described below.

\section{DESCRIPTION}

A plastic scintillator was chosen for this spectrometer because it is less expensive, easier to machine, and less fragile than organic crystals.* Fig. 1 shows the crosssections of the three detectors that were constructed. They were designed for maximum $\beta$-energies of $1.5,2.2$ and $3.6 \mathrm{MeV}$, respectively. Each detector is constructed in two parts consisting of a flat, solid, right circular cylinder used as the base, and a top piece in the form of a hollowed-out cylinder or truncated cone. The faces of the pieces are polished, and then the top optically connected to the base with silicone fluid.

The hollow detector is next wrapped with 1.5 mil aluminum foil except for the hole in the top and mounted on a photomultiplier tube as in Fig. 2. Each radioactive sample

* The particular plastic phosphor chosen was Sintilon, produced by National Radiac Company, Newark, N.J. It is similar in performance to trans-stilbene. 


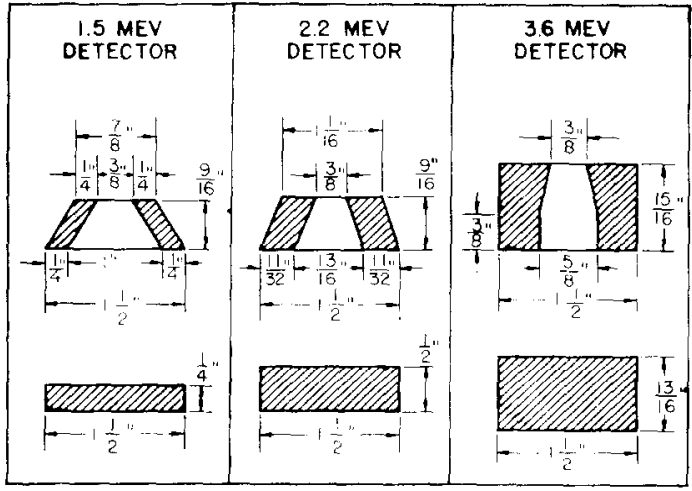

Fra. 1. Cross-sections of the three hollow plastic scintillators.

is mounted on 0.25 mil Mylar film coated on the reverse side with a thin layer of aluminum. The mounted sample is then covered with a 0.25 mil film of transparent Mylar and pressed tightly to the top of the detector over the entrance hole. The aluminum backing of the sample completes the light-tight reflector surrounding the detector. A rubber cap is finally placed over the detector and phototube housing to guard the detector from outside light.

Calibration of the spectrometer is accomplished using conversion electron peaks from

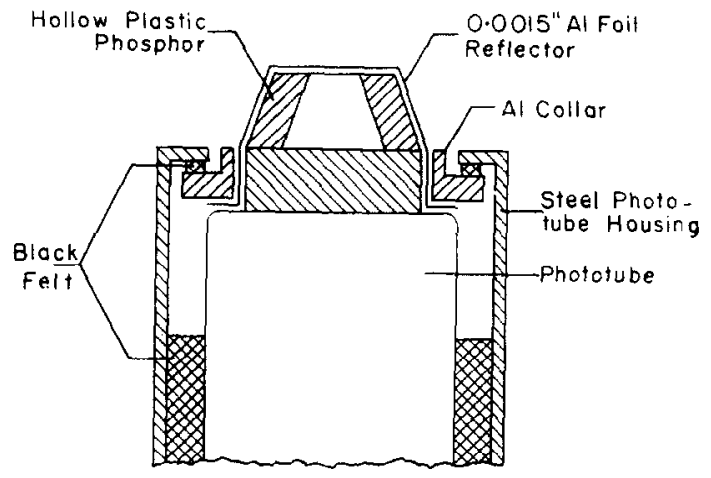

FIG. 2. Cross-section of $\beta$-ray detector mounting.

$\mathrm{In}^{114}, \mathrm{Sn}^{113}$ and $\mathrm{Cs}^{137}$, and also by measuring $\beta$-spectra with known maximum energy and shape. For the particular phosphor used here, the heights of the output pulses from the photomultiplier tube are linearly proportional to energy from about $0 \cdot 1 \mathrm{MeV}$ to at least $3.6 \mathrm{MeV}$. In the range from 0 to $0 \cdot 1 \mathrm{MeV}$ the calibration curve was approximated by continuing the calibration plot in a smooth curve down to the origin. A correction was made for the energy lost by the $\beta$-particles as they passed through the Mylar sample cover and through the air inside the detector. ${ }^{(8)}$

\section{CALGULATION}

It is, in general, more difficult to analyze a beta spectrum than a $\gamma$-spectrum, but since digital computers have become more available, this need no longer be a major problem. The spectra to be presented here have been analyzed ${ }^{(8)}$ on an IBM 650 computer where the average machine computation time was less than $\frac{1}{2}$ min per spectrum.

The shape of the $\beta$-spectral distribution may be represented by the following equation:

$$
\begin{array}{r}
N(W) d W \propto p W\left(W_{0} \quad \cdots W\right)^{2} F(Z, W) \\
{\left[M^{2}\right] d W .}
\end{array}
$$

Here $N(\boldsymbol{W})$ is the number of $\beta$-particles emitted with energy $W, W_{0}$ is the maximum beta energy, $p$ is momentum, $F(Z, W)$ is a Coulomb factor, and $M^{2}$ is a nuclear factor whose form depends upon whether the transition is allowed or forbidden. Rewriting equation (1), wc have

$$
\left[\begin{array}{c}
N(W) \\
p W F(Z, W)\left[M^{2}\right]
\end{array}\right]^{\frac{1}{2}} \propto W_{0}-W .
$$

A plot of the left hand side of equation (2) against energy $W$ is called a Kurie plot and yields a straight line intersecting the abscissa at $W_{0}$, the maximum $\beta$-energy. Tables of the function $p W F(Z, W)$ are available from the National Bureau of Standards. ${ }^{(9)}$ If the $\beta$-transition is allowed, $\left[M^{2}\right] \approx 1$ and is independent of energy. If the transition is forbidden, $\left[M^{2}\right]$ may be a complicated, energy-dependent function. Some of the Kurie plots discussed later are of forbidden transitions of a type that is particularly easy 
to handle. These are the "unique" firstforbidden transitions where

$$
\left[M^{2}\right] \equiv \alpha=\left(W^{2}-1\right)+\left(W_{0}-W\right)^{2} .
$$

The symbol $\sqrt{N / F}$ will be used in the subsequent figures to represent $\{N W /[p W F(Z$, $W)]\}^{1 / 2}$. The symbol $\alpha$ will be used to indicate that a correction for forbiddenness has been made.

The beauty of the Kurie representation is that a linear extrapolation of the plot will yield the maximum energy $W_{0}$, and also that it is a simple means of testing a spectrum for the allowed shape. If there is more than one beta transition present, the complex Kurie plot may be resolved to give a straight line for each component. The relative abundance of each $\beta$-ray group may then be determined from the area under each straight-line curve.

It is somewhat more convenient to use relativistic or rest mass energy units rather than $\mathrm{MeV}$ when correcting spectra for forbiddenness. The two units are simply related by the following equation.

$$
W=\frac{E}{m_{0} c^{2}}+1 \text {. }
$$

where $W$ is energy in relativistic units, $E$ is energy in $\mathrm{MeV}$, and $m c^{2}$ is the rest mass of the electron $(0.5109 \mathrm{MeV})$. When the transition is allowed, it is usually more convenient to substitute $E$ for each $W$ in equation (2).

\section{PERFORMANCE}

A number of corrections can be made to improve the relatively poor resolution of a scintillation $\beta$-ray spectrometer. In the following discussion the spectra have been corrected for resolution near their maximum

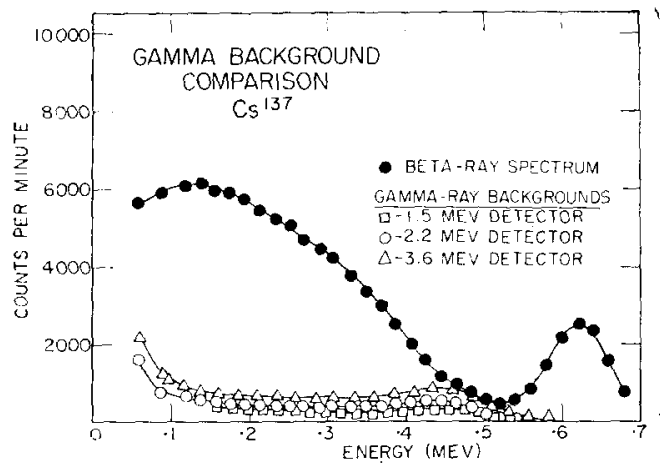

Fic. 3. $\gamma$-Ray backgrounds in the three plastic detectors.

energy endpoints only. (The resolution correction procedure is discussed at the end of this paper.)

$\gamma$-Ray background. The plastic $\beta$-ray detectors used here are also somewhat sensitive to $\gamma$-rays. Therefore, if $\gamma$-rays are present in a sample, the $\gamma$-background must be subtracted from the gross curve to get the $\beta$ spectrum. The $\gamma$-background may be obtained by placing an absorber in the entrance hole of the detector under the sample. The magnitude of this effect is shown in Fig. 3 where the backgrounds in each of the three previously described detectors have been normalized to a $\mathrm{Cs}^{137}$ $\beta$-ray spectrum. Assuming an equal number of $\beta$-rays and $\gamma$-rays in $\mathrm{Cs}^{137}$, the ratio of the $\gamma$ - to $\beta$-efficiencies in the usual experimental set-up is roughly 0.23 for the $3.6 \mathrm{MeV}$ phosphor, and 0.15 and 0.11 for the $2 \cdot 2$ and $1.5 \mathrm{MeV}$ phosphors, respectively.

Because of this background effect it is desirable to place the sample right at the entrance of the detector, rather than placing it a short distance away and using a collimator to direct the $\beta$-particles onto the bottom of the detector. The latter method not only does not improve the resolution, but also greatly increases the ratio of $\gamma$-rays to $\beta$-rays detected (assuming that the collimator does not appreciably reduce the number of $\gamma$-rays striking the detector).

Intercomparison of the three detectors. The performance of the $2 \cdot 2 \mathrm{MeV}$ detector is somewhat superior to the other detectors for $\beta$-rays up to $\sim 2 \mathrm{MeV}$. The reasons for this include the following:

(1) The much larger size of the $3.6 \mathrm{MeV}$ detector contributes to greater light scattering and a lower optical transmission.

(2) The optical interface between the top and bottom parts of an assembled detector is appreciably smaller in the case of the 
1.5 MeV detector than for the $2.2 \mathrm{MeV}$ detector.

These effects cause the resolution of the $2 \cdot 2 \mathrm{MeV}$ detector to be somewhat better than the others, as shown in Table 1. These

TABLE 1. Resolution of the three phosphors for the $\mathrm{Cs}^{137}$ conversion electron peak

\begin{tabular}{c|c}
\hline Phosphor & $\begin{array}{c}\text { Resolution uncorrected } \\
(\%)\end{array}$ \\
\hline $1.5 \mathrm{MeV}$ & 14.7 \\
$2.2 \mathrm{MeV}$ & 14.0 \\
$3.6 \mathrm{MeV}$ & 15.8 \\
\hline
\end{tabular}

resolutions have not been corrected for the higher energy $\beta$-ray present in $\mathrm{Cs}^{13}$, nor for the smearing of the low energy edge of the

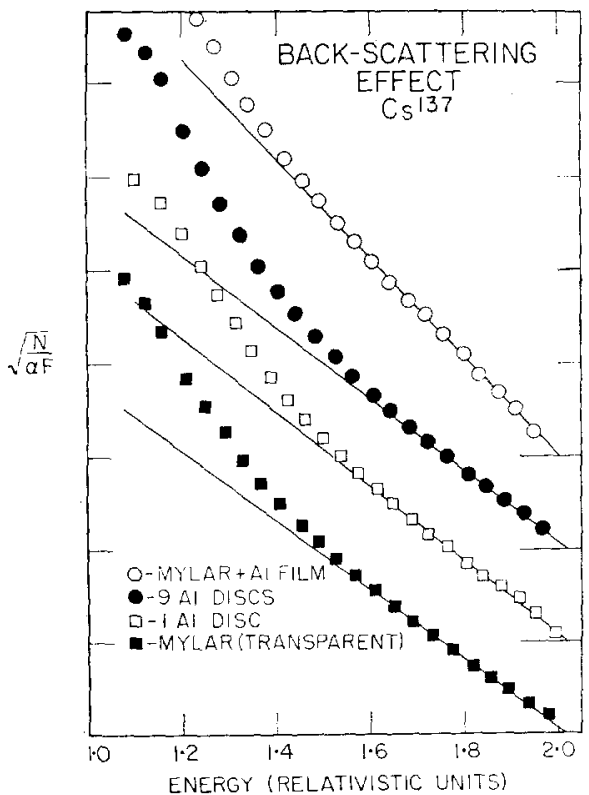

Frg. 4. Effect of sample backing on shape of the Kuric plot of $\mathrm{Cs}^{137}$.

peak. The true per cent resolution would be somewhat smaller in each case. Since a correction for resolution can be made, the advantage in using the $2 \cdot 2 \mathrm{MeV}$ detector is only slight.

Effect of sample backing. Essentially any amount of backing upon which the sample rests will cause some distortion of the $\beta$ spectrum due to backscattering. It is also of interest to compare light reflecting backings with transparent ones. Fig. 4 shows Kurie plots for $\mathrm{Cs}^{137}$ obtained using various sample substrates. The top curve is for the aluminum-coated Mylar normally used, and shows

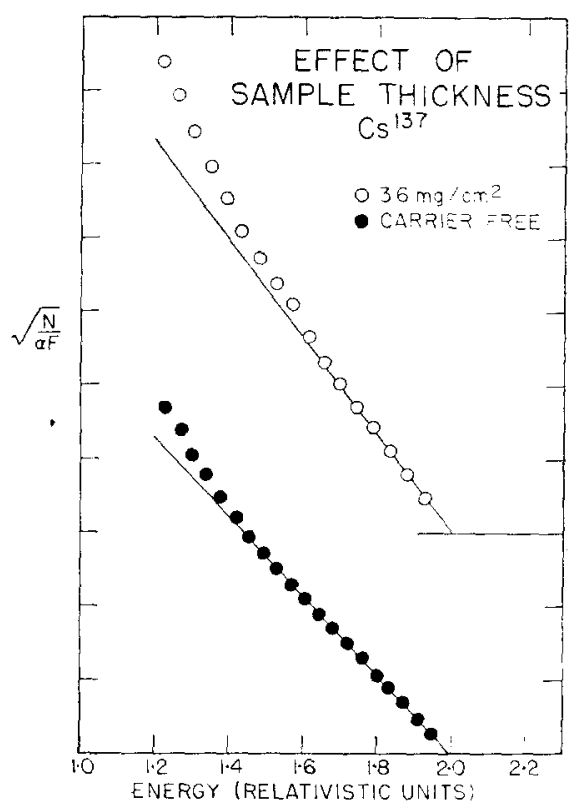

Fig. 5. Effect of sample thickness on the Kurie plot of $\mathrm{Cs}^{13}$.

much less distortion than the others. The bottom curve shows the results for $0.25 \mathrm{mil}$ transparent Mylar (no aluminum coating). The two center curves were obtained by placing first one and then nine $6 \mathrm{mil}$ aluminum discs behind the transparent Mylar backing. Further work along these lines showed that for most purposes it was not necessary to use extremely thin films (of the order of $\mathrm{mg} / \mathrm{cm}^{2}$ ) or sample mountings.

Thick samples. Occasionally carrier-free $\beta$ sources are not available, and the best sample that can be obtained is one of high specific activity. Since any weighable sample will tend to distort the $\beta$-spectrum, it is of interest to determine the magnitude of this distortion. In Fig. 5 a carrier-free source of $\mathrm{Cs}^{\mathbf{1 3 7}}$ is compared with a source made by mixing finely powdered $\mathrm{BaCO}_{3}$ with the 
$\mathrm{Cs}^{137}$ activity. The thick source had a surface density of $\sim 3.6 \mathrm{mg} / \mathrm{cm}^{2}$. While distorted, the thick sample still yields a usable Kurie plot over a portion of the range.

Forbidden spectra. A $\beta$-ray spectrometer must be accurate enough to distinguish between the shapes of allowed and forbidden spectra. Without this information the resolution of complex spectra may be extremely difficult if not impossible. To evaluate this

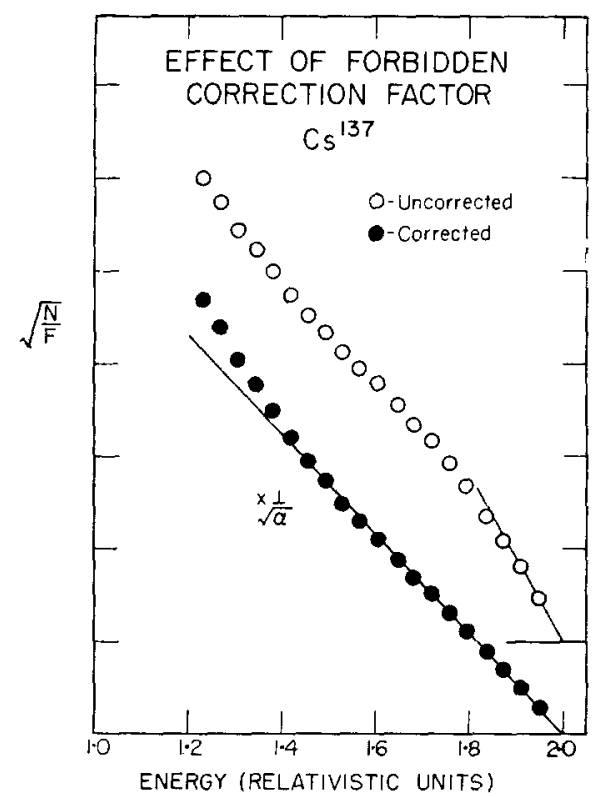

FIG. 6. Kurie plot $\mathrm{Cs}^{132}$ showing effect of forbidden correction term.

spectrometer a number of "unique" forbidden spectra were examined Fig. 6 shows the results for $\mathrm{Cs}^{137}$. The pronounced difference in shape, i.e. the skewing of the uncorrected spectrum toward higher energies is typical of forbidden spectra.

Complex spectra. If there is more than one beta emitter present in a sample, it may be of interest to determine the relative abundance of each emitter. Fig. 7 shows the Kurie plot obtained when a $\mathrm{Cs}^{137}$ and a $\mathrm{Pm}^{147}$ sample were examined simultaneously. After the $\mathrm{Cs}^{137}$ component was subtracted, the allowed Kurie plot for $\mathrm{Pm}^{147}$ could be calculated. This resolved curve is presented in Fig. 8. The area under each Kuric plot was com-

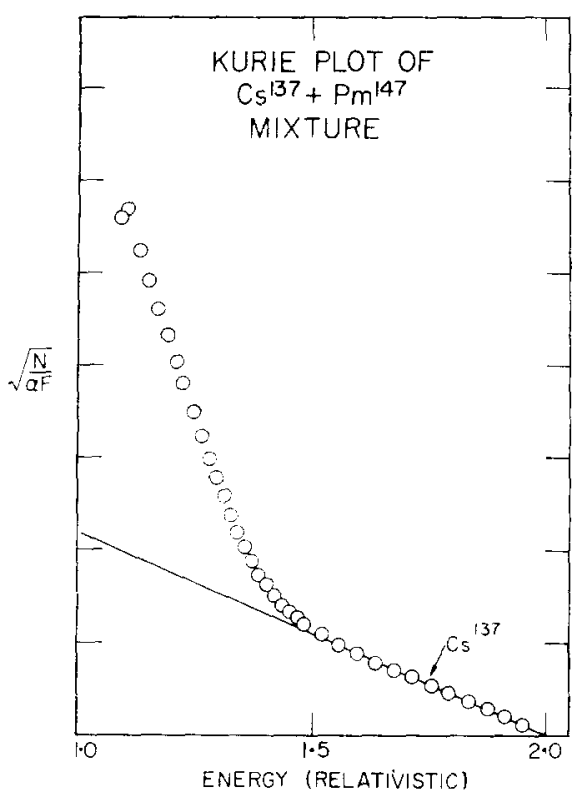

FIG. 7. Kurie plot of $\mathrm{Cs}^{137}+\mathrm{Pm}^{147}$ mixture.

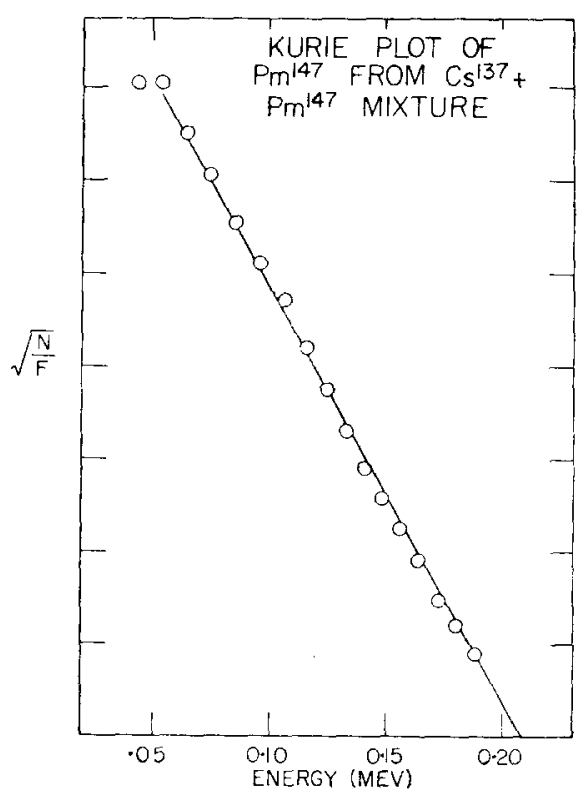

FIG. 8. Kuric plot of $\mathrm{Pm}^{147}$ resolved from $\mathrm{Cs}^{137}+\mathbf{P m}^{147}$ mixture.

pared with the Kurie plots taken individually on each sample. The ratio of the areas in the mixture was within 4 per cent of the ratio from the individual samples. 
The apparent straightness of the $\mathrm{Pm}^{147}$ Kurie plot, down to $\sim 0.05 \mathrm{MeV}$, is somewhat accidental. The Kurie plot obtained on the $\mathrm{Pm}^{147}$ sample alone was straight down to $\sim 0.08 \mathrm{MeV}$ where the observed points began to fall below the straight line. The

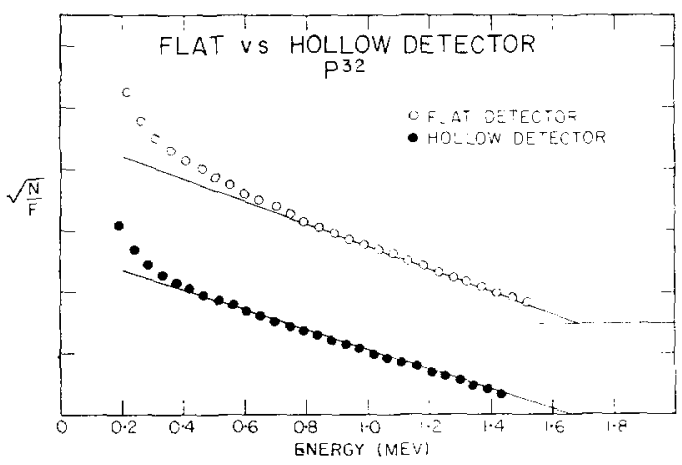

FIG. 9. Kuric plots for $\mathrm{P}^{32}$ from both flat and hollow detcctors.

upward curvature of the $\mathrm{Cs}^{137}$ Kurie plot at low energies was not taken into account when it was subtracted from the plot of the mixture. Hence the resolved $\mathrm{Pm}^{147}$ curve contained a $\mathrm{Cs}^{137}$ component which obscured the true shape at low energies. Correction for instrument resolution over the entire mixed spectrum would have eliminated much of this difficulty, and would have produced more accurate results for the ratio of the components.

Comparison with flat detectors. A comparison was made between the performance of the hollow detector and a flat detector. The base of the $2 \cdot 2 \mathrm{MeV}$ hollow detector was covered with 1.5 mil aluminum foil, and was used as the flat detector with the source $2 \cdot 2 \mathrm{~cm}$ from the detector along its axis. The resolution for the $\mathrm{Cs}^{137}$ conversion electron peak was 24 per cent for the flat detector and 14 per cent for the $2 \cdot 2 \mathrm{MeV}$ hollow detector. Conversion peak to valley ratios were $4 / 1$ and $6 / 1$ for the flat and the hollow detectors, respectively. The improvement in resolution by a factor of more than 1.7 is important since the spectra are thereby less distorted and resolution correction over the entire spectrum may not be necessary.
Fig. 9 shows the allowed Kurie plot for P32 obtained on each detector. While the flat detector produces a Kurie plot that is linear over about half the range, the plot

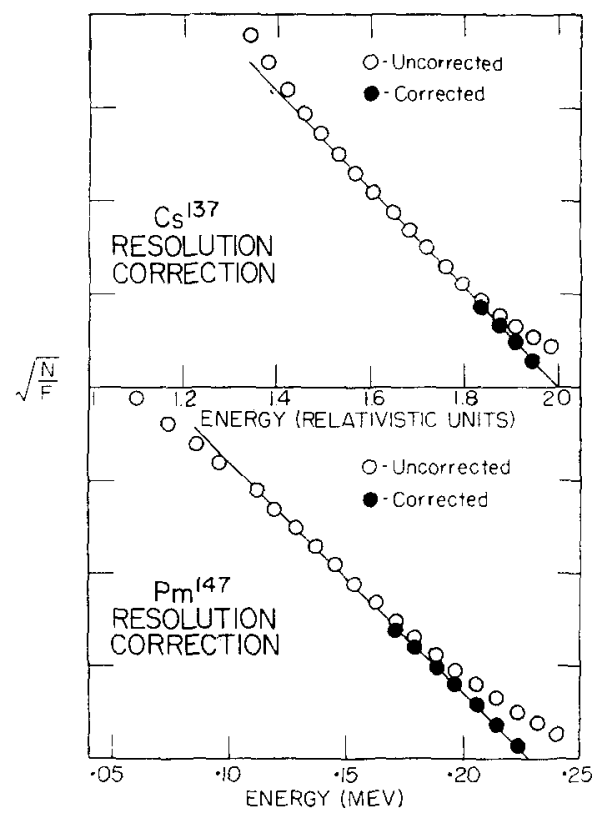

FIG. 10. Effect of resolution correction near the endpoint of $\beta$-spectra.

from the hollow detector is linear over more than three-quarters of the range. On both curves the sharp upward curvature at the lower end is due in part to $\mathrm{P}^{33}$ in the sample.

Correction for instrument resolution. Because of the low resolving power of scintillation $\beta$-ray spectrometers, the observed spectral shape does not correspond exactly to the true shape. In fact, at both the low energy and the high energy ends, the distortion usually proves to be very significant. The experimentally measured spectrum $M(W)$ is related to the true spectrum $N(W)$ by the following equation:

$$
\begin{aligned}
M(W) & =\int_{0}^{W_{0}} N(W) S(W) d W \\
& \simeq \Sigma_{i} N\left(W_{i}\right) S\left(W_{i}\right) \Delta_{i} W
\end{aligned}
$$

where $W$ is energy, $W_{0}$ is the maximum $\beta$ energy, and $S(W)$ is the so-called instrument profile or line shape.

Several articles $(2,10,11,12)$ have appeared discussing the correction for instrument 
resolution assuming a Gaussian distribution for the instrument profile. This works well enough at the high energy end, but uniformly

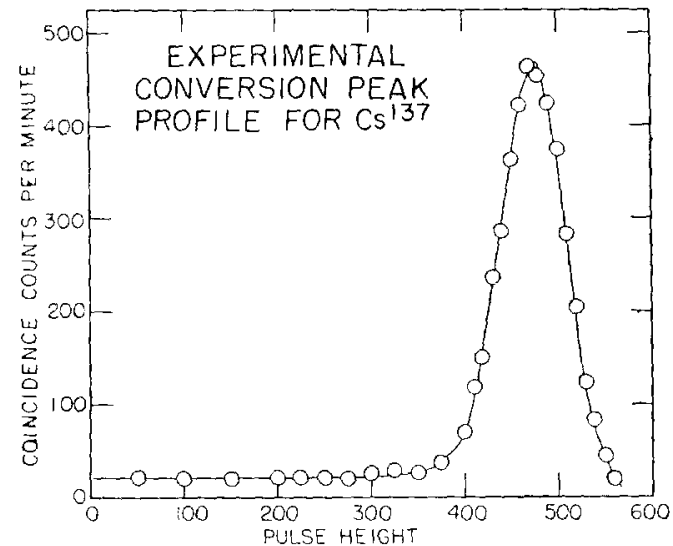

FIG. 11. Experimental conversion peak profile for $\mathrm{Cs}^{137}$.

fails to eliminate upturns at low energy. Fig. 10 shows the effect of this type of correction near the maximum energy using the method of PALMER and LASLETT. ${ }^{(2)}$

Perhaps the best approach to the resolution problem was put forth by FREEDMAN et al. ${ }^{(13)}$ in June, 1956. They assumed that the instrument profile was not symmetrical. Following their approach and using coincidence methods, the conversion electron peak of $\mathrm{Cs}^{137}$ was measured on the $2 \cdot 2 \mathrm{MeV}$ hollow detector and is shown in Fig. 11. It can be seen that the curve extends all the way down to zero energy at a constant value of 5 per cent of the peak height. Using this line shape the Kurie plot for $\mathrm{Cs}^{137}$ was corrected and is shown in Fig. 12. Now the Kurie plot is straight down to $\sim 0.05 \mathrm{MeV}$.

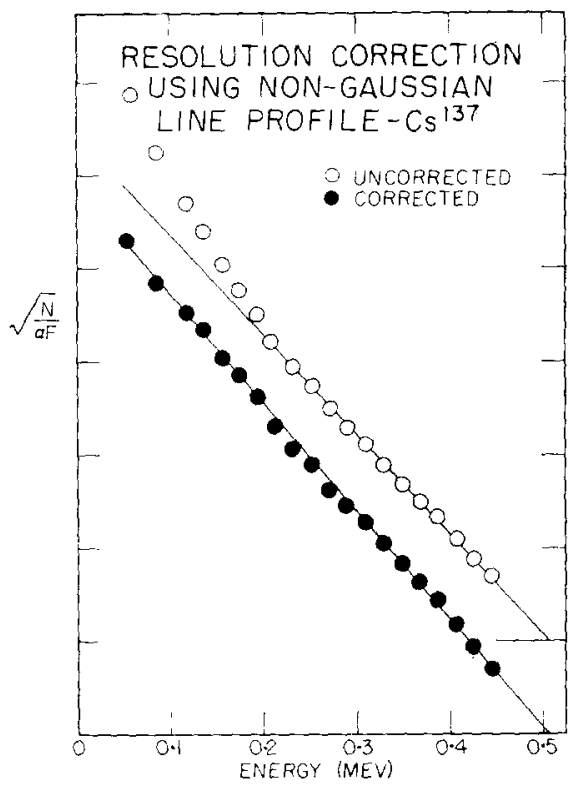

FIG. 12. Effect of non-Gaussian resolution correction on $\mathrm{Cs}^{197}$ Kurie plot.

Acknowledgements--The authors are indebted to Mrs. JeAnNe C. Gardner for her work in programming the Kurie analysis for the IBM 650 computer.

This work was supported in part by the U.S. Atomic Energy Commission.

This paper was presented before the Second Industrial Nuclear Technology Conference, Chicago, Illinois on May 15, 1957.

\section{REFERENCES}

1. Hunt W. A. and Zaffarano D. J. AEC Report ISC-469 (1954).

2. Palmer J. P. and Laslett L. J. AEC Report AEGU-1220 (1951).

3. Siegbahn K. (editor) Beta- and Gamma-Ray Spectroscopy pp. 132-164. Interscience, New York (1955).

4. Jordon W. H. Annu. Rev. Nucl. Sci. 1, 207-244 (1952).

5. Oak Ridge National Laboratory AEC Report ORNL-940 (1950).

6. Oak Ridge National Laboratory AEC Report ORNL-1092 (1951).

7. O'Kelly G. D., Lazar N. H. and Eichler E. Phys. Rev. 101, 1059 (1956).
8. Gardner D. G., Ph.D. Thesis, University of Michigan (May 1957).

9. Tables for the Analysis of Beta Spectra. National Bureau of Standards, Applied Mathematics Series 13 (1952).

10. Bracewell R. N. F. Opt. Soc. Amer. 45, 873-876 (1955).

11. Ketelle B. H. Phys. Rev. 80, 758 (1950).

12. Owen G. E. and Primakoff H. Phys. Rev. 74, 1406 (1948).

13. Freedman M. S. et al. Rev. Sci. Instrum. 27, 716 (1956). 